\title{
Study of Salivary Enzymes in Oral Lesions
}

\author{
Reshma P Chavan ${ }^{1}$, Shivraj M Ingole ${ }^{2}$, Shubhangi M Dalvi ${ }^{3}$
}

\begin{abstract}
Aim and objective: Saliva contains different enzymes, which reflect metabolic activities of the body. Certain enzymes, such as, aspartate aminotransferase (AST) and alanine aminotransferase (ALT) enzymes are products of cell injury and cell death. Some benign oral conditions having malignant potential and therefore early changes in salivary enzyme level may be helpful for the early detection of malignancy. An attempt is made to compare salivary enzyme levels (AST and ALT) in benign oral lesions in tobacco addicts, malignant oral lesions in tobacco addicts, and healthy non-tobacco addict patients with age- and sex-matched control group.

Material and methods: A retrospective study of 150 patients with benign and malignant oral lesions with tobacco addiction was done. Control group includes tobacco non-addicts. The oral lesion was noted. Biopsy of oral lesion was sent to histopathology for diagnosis. The saliva of all patients was collected for salivary enzymes (AST and ALT) examination. Levels of salivary enzymes (AST and ALT) in benign and malignant oral lesions of patients with tobacco addiction and tobacco non-addict control group without any oral lesions with age- and sex-matched study group were included in the study. The results were analyzed statistically by Student's t-test.

Results: Salivary enzymes (AST and ALT) were not significantly increased in benign and malignant oral lesions in tobacco addict patients.

Conclusion: Salivary enzymes were increased in all benign and malignant oral lesions in tobacco consumers. There was no significant increase in salivary enzymes in all benign and malignant oral lesions in tobacco consumers.

Clinical significance: Research is going on salivary biomarkers, but these salivary biomarkers lack sensitivity and specificity and cannot be applied. Keywords: Benign, Malignant, Oral lesion, Salivary enzymes.

Otorhinolaryngology Clinics: An International Journal (2020): 10.5005/jp-journals-10003-1348
\end{abstract}

\section{INTRODUCTION}

Tobacco consumption leads to staining of teeth and dental plagues and is a significant risk factor for oral diseases. Though the bases of benign and malignant oral lesions are multifactorial, it definitely reflects metabolic changes in the body due to cell death and cell injury. Changes in the constituents of saliva are associated with certain diseases and therefore helpful for diagnosis. ${ }^{1,2}$ Level of enzymes, such as, peroxidase, lactate dehydrogenase, creatinine kinases, aspartate aminotransferase (AST), and alanine aminotransferase (ALT) is increased in certain diseases. In oral cavity diseases, these enzyme levels are increased in saliva. It has been studied that salivary levels of AST and ALT were increased in periodontitis, and after treatment, these enzyme levels get decreased. ${ }^{3}$ According to Dewan et al., AST level in GCF could be a potential biochemical marker for periodontal disease progression. ${ }^{4}$ The AST and ALT values in the saliva were 138 to $229 \%$ of normal value in all head and neck cancers. ${ }^{5}$ Thus, in all oral diseases, AST and ALT enzymes in the saliva were increased. The values of these enzymes decrease or come to normal levels at the end of treatment. In $92 \%$ of head and neck cancer cases, the AST and ALT were normal or near-normal after radiotherapy. Therefore, salivary enzymes, such as, AST and ALT can be used as biochemical tumor markers in malignant conditions. Rai et al. showed that salivary AST, ALT, and LDH levels can be used as markers of oral diseases. ${ }^{6}$ Oral malignancy can be detected early with the help of salivary biomarkers. ${ }^{7}$ It may also be possible to detect early oral malignancy with the help of salivary levels of cytokines. ${ }^{8}$

\section{Materials and Methods}

A retrospective study of 150 patients with benign oral lesion was included in this study. Tobacco addict patients with benign and malignant oral lesions among 15 years and 60 years of the age
${ }^{1}$ Department of ENT, Dr. Vaishampayan Memorial Government Medical College, Solapur, Maharashtra, India

${ }^{2}$ Department of Radiology, GGMC and Sir JJ Group of Hospitals, Mumbai, Maharashtra, India

${ }^{3}$ Department of Biochemistry, GGMC and Sir JJ Group of Hospitals, Mumbai, Maharashtra, India

Corresponding Author: Reshma P Chavan, Department of ENT, Dr. Vaishampayan Memorial Government Medical College, Solapur, Maharashtra, India, Phone: +919870569697 e-mail: entproblem@ gmail.com

How to cite this article: Chavan RP, Ingole SM, Dalvi SM. Study of Salivary Enzymes in Oral Lesions. Int J Otorhinolaryngol Clin 2020;12(1):1-3.

Source of support: Nil

Conflict of interest: None

group who attended ENT OPD in medical college hospitals in Mumbai from January 2013 to June 2014 were selected. In the present study, tobacco addict patients were selected on personal interview. Daily tobacco consumption in any one or more form of tobacco, tobacco with lime, tobacco consumption in the pan (quit), tobacco mishari application on teeth, tobacco smoking in the form of bidis and cigarettes, ghutka, mawa, jerda, tobacco used as snuff, etc., for more than two years duration was included in tobacco addiction. The selection of patients was done at random. Patients with salivary gland tumors and diseases and patients below 15 years. The age was excluded from the study. Patients with diabetes mellitus and known hormonal disorders were also excluded from the study. Healthy persons without tobacco addiction and without any oral lesion whose age and sex were matched were included 
in the control group. Written informed consent was taken from the participants.

The study was approved by the Ethics Committee of Institute.

Collection of saliva samples $(5 \mathrm{~mL})$ was done using the "draining method" from the mouth of the patient. Saliva was collected in sterile containers, and the saliva samples were centrifuged at 10,000 rpm for 10 minutes. AST and ALT in the collected samples were determined spectrophotometrically.

Statistical analysis was done to compare the enzymatic activity among the tobacco chewers with the benign and malignant oral regions and healthy non-tobacco consumers.

\section{Results}

Table 1 shows AST and ALT among healthy subjects, in control, benign, and malignant oral conditions. The results showed that AST in patients with benign and malignant oral conditions was higher than the control group, and it is statistically not significant. Salivary enzymes, such as, ALT in patients with benign conditions were slightly lower and in malignant oral conditions were higher than the control group. But the results were statistically not significant.

The obtained results have shown that AST and ALT in the saliva of the patients with benign and malignant conditions were not significantly increased as compared with the control group.

\section{Discussion}

There are salivary glands present in the human body. According to the size of glands, these glands are divided into two groups, major and minor salivary glands. Parotid glands, submandibular glands, and sublingual glands are three major salivary glands. Secretions of these glands contain water, electrolytes, sodium, potassium, calcium, chloride, magnesium, bicarbonate, and phosphate. It also contains proteins, such as, enzymes, mucosal glycoproteins, albumin, some polypeptides, oligopeptides, and immunoglobulin. Glucose, urea, and ammonia are also seen in salivary gland secretions. Salivary enzymes are proteins. These are a-amylase, albumin, cystins, hystatins, secretory $\lg \mathrm{A}$, and lactoferrin. ${ }^{9}$ Some proteinous and non-proteinous substances require advanced instruments for their detection. CA125, haptoglobin, profilin-1, and transferrin are salivary markers for breast carcinomas. ${ }^{10,11}$ Salivary marker for lung cancer is $\$ 100$ calcium-binding protein A9. ${ }^{12}$ Salivary annexin A1, carbonic anhydrase $\mathrm{VI}$, S100 calcium-binding protein, and lipocalin are helpful for the detection of both breast and lung cancers. ${ }^{13}$ Cox multivariate analysis showed that for RFS, Stat3 and c-myc, and for OS, Stat3, Bcl-2, and p53 were helpful for the prognosis of tongue carcinoma. Buccal mucosa and tongue carcinoma have different salivary markers. ${ }^{13}$

A study done by Girja et al. in 2002 has proven major changes in the salivary biochemical composition of leukoplakia (premalignant) and oral malignancy. ${ }^{14}$ These changes may be due to tobacco uses or disease process. Brailo et al. ${ }^{15}$ also studied changes in the

Table 1: AST and ALT in control, benign, and malignant ENT lesions

\begin{tabular}{lcc}
\hline Mean (SD) & \multicolumn{1}{c}{ AST } & \multicolumn{1}{c}{ ALT } \\
\hline Control & $4.8 \pm 3.53$ & $7.71 \pm 1.41$ \\
Benign & $6.23 \pm 0.25$ & $5.84 \pm 0.4$ \\
Malignant & $44.75 \pm 9.19$ & $26.32 \pm 9.90$ \\
\hline
\end{tabular}

salivary IL- 6 and tumor necrosis factor alpha (TNF-a) in benign and malignant conditions.

In the present study, there was a rise in salivary AST in a study group, which was not significant. There was no significant change in salivary AST between benign and malignant conditions and control group. Research is going on salivary biomarkers, but these salivary biomarkers lack sensitivity and specificity and cannot be applied.

\section{Conclusion}

In the present study, there was a rise in salivary AST in a study group, which was not significant. There was no significant change in salivary AST between benign and malignant conditions and control group.

\section{Clinical Significance}

Research is going on salivary biomarkers, but these salivary biomarkers lack sensitivity and specificity and cannot be applied.

\section{ACKnowledgment}

The authors are thankful to Dr VW Patil, Professor and Head, Department of Biochemistry, GGMC and Sir JJ Group of Hospitals, Mumbai, India, for his encouragement to prepare the study material.

\section{References}

1. Shah FD, Begum R, Vajaria BN, et al. A review on salivary genomics and proteomics biomarkers in oral cancer. Indian J Clin Biochem 2011;26:326-334. DOI: 10.1007/s12291-011-0149-8.

2. Wong DT. Salivary diagnostics for oral cancer. J Calif Dent Assoc 2006;34:303-318.

3. Dabra S, China K, Kaushik A. Salivary enzymes as diagnostic markers for detection of gingival/periodontal disease and their correlation with the severity of the disease. J Indian Soc Periodontol 2012;16:358364. DOI: 10.4103/0972-124X.100911.

4. Dewan A, Bhatia P. Evaluation of aspartate aminotransferase enzyme levels in saliva and gingival crevicular fluid with periodontal disease progression-A pilot study. J Int Oral Health 2011;3:19.

5. Chougule A, Hussain S, Agarwal DP. Prognostic and diagnostic value of serum pseudocholinesterase, serum aspartate transaminase and serum alinine transaminase in malignancies treated by radiotherapy. J Cancer Res Ther 2008;4:21-25. DOI: 10.4103/0973-1482.39601.

6. Rai B, Kharb S, Anand SC. Salivary enzymes and thiocynate: salivary markers of periodontitis among smokers and non-smokers; a pilot study. Adv Med Dent Sci 2007;1:1-4.

7. Rhodus NL, Ho V, Miller CS, et al. NF-kappaB dependent cytokine levels in saliva of patients with oral preneoplastic lesions and oral squamous cell carcinoma. Cancer Detect Prev 2005;29:42-45. DOI: 10.1016/j.cdp.2004.10.003.

8. Rhodus NL, Cheng B, Myers S, et al. The feasibility of monitoring NF-kappaB associated cytokines: TNF-alpha, IL-1alpha, IL-6, and IL-8 in whole saliva for the malignant transformation of oral lichen planus. Mol Carcinog 2005;44:77-82. DOI: 10.1002/mc.20113.

9. Cheng $Y$, Wright J. Advances in diagnostic adjuncts for oral squamous cell carcinoma. Open Pathol J 2011;5:3-7. DOI: 10.2174/1874375701105010003.

10. Chiappin S, Antonelli G, Gatti R, et al. Saliva specimen: a new laboratory tool for diagnostic and basic investigation. Clin Chim Acta 2007;383:30-40. DOI: 10.1016/j.cca.2007.04.011.

11. Agha-Hosseini F, Mirzaii-Dizgah I, Rahimi A, et al. Correlation of serum and salivary CA125 levels in patients with breast cancer. J Contemp Dent Pract 2009;10:E001-E008. 
12. Xiao $H$, Zhang $L$, Zhou $H$, et al. Proteomic analysis of human saliva from lung cancer patients using two-dimensional difference gel electrophoresis and mass spectrometry. Mol Cell Proteomics 2012;11:M111.012112. DOI: 10.1074/mcp.M111.012112.

13. Bigler LR, Streckfus CF, Dubinsky WP. Salivary biomarkers for the detection of malignant tumors that are remote from the oral cavity. Clin Lab Med 2009;29:71-85. DOI: 10.1016/j.cll.2009.01.004.
14. Girja KP, Sundharam BS, Krishnan PA, et al. Biochemical changes of saliva in tobacco chewers tobacco smokers, alcohol consumers, leukoplakia and oral cancer patients. Indian J Dent Res 2002;13:102-107.

15. Sato J, Goto J, Murata T, et al. Changes in saliva interleukin- 6 levels in patients with oral squamous cell carcinoma. Oral Surg Oral Med Oral Pathol Oral Radiol Endod 2010;110:330-336. DOI: 10.1016/j. tripleo.2010.03.040. 